\title{
Research on Solving Ill-Structured Problems for e-Learning: Cognitive Perspectives
}

\author{
Jianzhen Chen and Xiaoping Li
}

\begin{abstract}
Ill-structured problems are defined as having vague goals that permit multiple solution paths. Solving ill-structured problems requires domain-specific knowledge as well as structural knowledge. Domain-specific knowledge is content knowledge consisting of cognitive components such as propositional information, concepts, rules, and principles.This article focuses on how to promote ill-structured problems solving. Using maps may solve ill-structured problems associated with visualizing information structure. Activities have been found to be effective in facilitating ill-structured problems-solving processes.Metacognition plays a key role in solving it. Advance organizers will promote connections between prior knowledge and the to-be-learned ill-structured problems in e-learning. It is necessary to reduce extraneous cognitive load caused by interactivity
\end{abstract}

Index Terms-Ill-structured problems, concept maps, meta-cognition, advance organizers, cognitive load.

\section{INTRODUCTION}

Many problems encountered in our professional lives pose uncertainties in various ways, including the complexity of the problems context; multiple and, often conflicting, perspectives among different stakeholders; diverse solutions or no solution; and multiple criteria for solution evaluation. These are the general features of ill-structured problems.

Ill-structured problems are defined as having vague goals that permit multiple solutions or solution paths. By contrast, well-structured problems have single solutions, optimal solution paths, and structured goals. Solving well-structured problems normally involves representing the problems, searching for solutions, and implementing solutions. However, because of the nature of an ill-structured problem, its solution process is different from that of a well-structured problem [1]. Problems representation, justification skills, monitoring, and evaluation are the primary requirements for ill-structured problems solving.

As opposed to well-structured problems, ill-structured problems usually have the following features [1].

- The goal is vaguely stated, and requires analysis and refinement in order to make the particular issue tractable.

- The constraints of the problems typically are not in the problems statement; instead, the solver needs to retrieve and examine the constraints when appropriate during the solving process.

- In most cases, the solver's solution is divided into a

Manuscript received June 6, 2014; revised August 18, 2014.

Jianzhen Chen and Xiaoping $\mathrm{Li}$ are with the Beijing Institute of Technology and Tianjin University of Technology and Education, China (e-mail: jzhen22@126.com). representation and a solution phase, as previously discussed. However, in contrast to well-structured problems, different solvers may vary considerably in the nature and contents of each of the phases. This is because ill-structured problems may be approached in different ways, according to the solder's knowledge, beliefs, and attitudes.

- Solutions to ill-structured problems typically are not right or wrong, and not valid or invalid; instead, solutions usually are regarded in terms of some level of plausibility or acceptability. Furthermore, solution evaluation may be a function of the evaluator's knowledge and beliefs regarding the issue at hand.

We argue that learners can be effectively supported by appropriate question prompts that direct their attention on important contextual issues in the cases they study. These prompts can be considered as processing prompts, meaning that they aim to stimulate learners' cognitive processing of the material in specific ways.

There are three cognitive processes: perception, memory recall, and reasoning.

- Perception-induced refers to contextual information available through perception of the environment. When studying cases, learners need to focus on crucial events that have an impact on the outcome of the case. By studying several concrete problem cases, learners are expected to construct abstract schemata that help them discriminate between good and bad decisions in the field of practice.

- Memory-induced refers to elements that are recalled from memory and older representations, which are reactivated. In order for learners to construct efficient schemata, it is important to comparatively process information from new cases with regard to what is already known from previous ones. Thus, memory-induced context becomes a point of reference for new information and supports the continuous reshaping of case-based cognitive schemata.

- Reasoning-induced refers to representations that are derived through reasoning processes. Learners need to reason based on newly established schemata.

\section{THEORETICAL BACKGROUND}

Knowledge must be understood as a dynamic system, constantly changing and reshaping. Knowledge is not a copy of stable reality, but an active construction of dynamic reality comprised of an interconnected web of patterns. When knowing is conceptualized as a highly complex, nonlinear phenomenon, two learners with slightly different initial knowledge will be expected to arrive at totally different 
learning outcomes after the much iteration that makes up knowing. Small differences in learning and the social, political, and economic context of schools may lead to dramatic changes in individual student outcomes, even within a single lesson. When a large number of explicit and hidden variables from an independent network exists, the learning process may become convoluted and imbalanced to the point that learning appears chaotic. New knowledge destabilizes the learning process, thus necessitating reorganization and restabilization [2]. The self-organizing nature of this dynamic process facilitates the evolution of increasingly sophisticated thought processes that respond to the complexity of the learning environment [3].

Though powerful as a cognitive processing tool, the working memory is, however, inhibited by its limited capacity and limited duration. It could be easily overloaded if more than a few chunks of new information are processed simultaneously.

Processing limitations of working memory (WM) impose severe restrictions on changes to long-term memory (LTM) knowledge base that should necessarily be incremental and slow. Even though we borrow most components of our vast knowledge base from other sources rather than discover or build them from the scratch, we always actively reconstruct this knowledge within our WM. The borrowed elements of information are reorganized and integrated with available prior knowledge structures from LTM. In situations where information cannot be borrowed from other sources, we use our default problem-solving mechanisms based on search for possible solution steps followed by tests of their effectiveness. Since these processes occur within limited WM, they impose a heavy cognitive load and leave minimal, if any, cognitive resources for meaningful construction of organized knowledge structures in LTM, thus providing inadequate conditions for learning.

\section{INFLUENCING FACTORS OF SOLVING ILL-STRUCTURED PROBLEMS}

There are many factors of influencing ill-structured problems. But learners ' cognitive, tool, activity and resource play an important role in e-learning. Four factors are depicted in Fig. 1

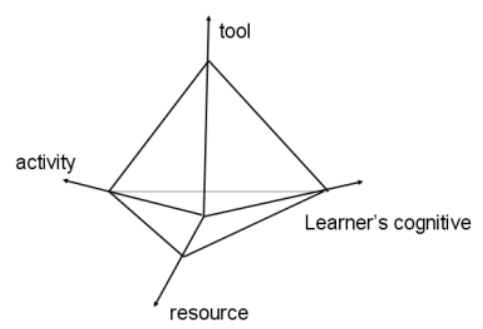

Fig. 1. Influencing factors of solving ill-structured problems.

\section{How to Promote Ill-StRUCTURED PROBLEMS SOLVING}

\section{A. Learner's Cognitive}

First, it is important for learners to use their metacognition. Solving ill-structured problems requires domain-specific knowledge as well as structural knowledge. Domain-specific knowledge is content knowledge consisting of cognitive components such as propositional information, concepts, rules, and principles. Structural knowledge is knowledge of how concepts within a domain are interrelated and requires integration of declarative knowledge into useful knowledge structures.

However, in the absence of domain-specific knowledge and structural knowledge, metacognition, which involves both knowledge and regulation of cognition, is necessary for solving ill-structured problems. Successful learners tend to generate more working explanations, particularly in response to an awareness of limited understanding. Metacognitive knowledge can compensate for absence of relevant domain knowledge when metacognitive awareness leads to recognizing areas of limited understanding, adopting working hypotheses, asking questions, monitoring thinking, and revisiting early interpretations.

In Flavell's model self-assessment plays a key role in his conception of metacognition.Metacognitive skills are generally divided into two types: self-assessment (the ability to assess one's own cognition) and self-management (the ability to manage one's further cognitive development). According to the research on self-assessment, learners who are skilled in metacognitive self-assessment and are therefore aware of their abilities are more strategic and perform better than those who are unaware.

In relation to metacognitive knowledge, the accuracy of self-knowledge - that is, having accurate perceptions and making accurate judgments about one's knowledge and skills - is relevant to learning.

In addition, reducing extraneous cognitive load caused by interactivity

To reduce the influence of missing prior knowledge on the effectiveness of interactive learner-controlled environments, Moreno and Mayer suggested including pre-training sessions that would activate or provide learners with relevant prior knowledge (the pre-training principle). For relatively advanced learners, the efficient interactive nonlinear exploration in complex ill-structured domains (e.g., literature or history) could be based on traversing the information space along several pre-defined intersecting dimensions and revisiting the same content material in a variety of different contexts [1]. With this method, learners are effectively provided overlapping instructional sub-goals that may prevent irrelevant random search activities that could otherwise overload WM capacity. Interestingly, for advanced learners, such partially directed exploration in complex unstructured interactive environments might have cognitive load consequences similar to eliminating specific problem-solving goals for novice learners in simple well-structured domains.

\section{B. Tools: Concept Maps}

Concept mapping is considered to be essential for a constructivist approach to learning and problems solving. The idea behind all methods and tools is that cognitive processing of complex subject matter may be enhanced if the task-relevant knowledge is well-organized and the structures behind ideas, knowledge and information, as well as their 
relevance for comprehension, learning and problems solving, are made explicit by means of visualization. Supporting just-in-time access to task-relevant information resources may help users in coping with task situations when they need additional data and information for cognitive processing.

Concept maps in e-learning have the potential to foster spatial learning strategies by visualizing the knowledge and support processes of individual knowledge management, such as the acquisition, organization, representation, evaluation, communication, localization, and utilization of knowledge[3], [4].

Mapping tools (e.g. concept mapping and mind mapping tools) in e-learning have the further potential to represent the content knowledge of a domain in a multi-media format, as well as knowledge about information resources.

Concept mapping tools in e-learning may contribute to the development of spatial strategies. Representing externally what is known about the subject matter or learning content may help learners to organize and represent conceptual and content knowledge about a domain in an integrative manner and to link individual domain knowledge to task-relevant information resources. These features enable concept mapping tools to be used as cognitive tools for managing knowledge and information [3].

Advanced computer-based concept mapping tools provide facilities for representing and accessing different kinds of knowledge: conceptual knowledge, content knowledge, as well as information resource knowledge. Content knowledge and information resources may be directly accessed from a concept node by just clicking on an icon associated with the node or on a hyperlink connecting the abstract concept with the related resource.

Concept Maps may be used to coherently represent in virtual space abstract conceptual knowledge, content knowledge, and related information. They may particularly be useful to supplement visualizations of information which are based on visual semantics only, and may help to make sense of the semantic relations between knowledge and information.

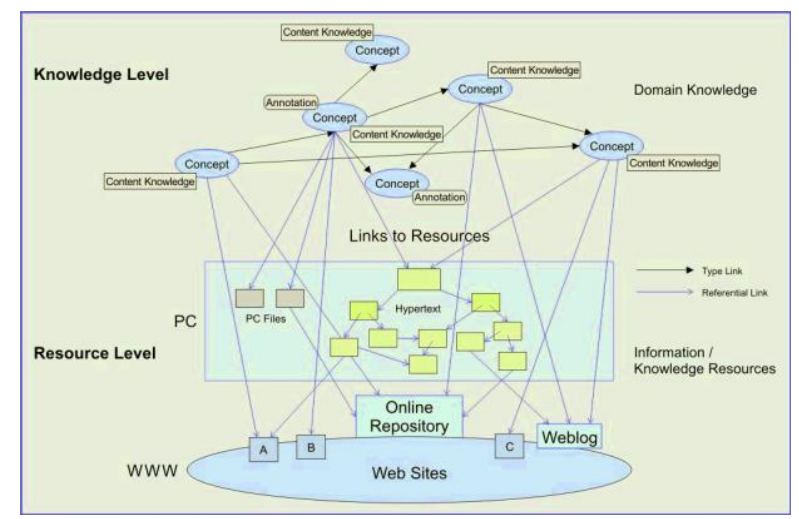

Fig. 2. Links of concept map to knowledge.

Links of concept map to knowledge is depicted in Fig. 2. On the one hand, the model visualizes the relation of conceptual knowledge, content knowledge and information resources. On the other hand, it shows how advanced digital concept mapping tools can simultaneously enable a knowledge-based visual organization, search and access of conceptual knowledge, content knowledge, and related information. There is a great deal of evidence indicating that the use of concept maps is a valuable strategy for supporting cognitive processing in a variety of learning and instructional settings, idea and knowledge generation, self-assessment of understanding and knowledge, collaborative work in distributed cognitive tasks.

Concept maps as graphic displays seem to permit problems solving by facilitating perceptual judgments of a kind that are almost effortless for humans, and can act as aids to retrieval. They help to exploit the rapid processing capabilities of the human visual system" and "very easy perceptual judgments are substituted for more difficult logical ones. An additional cognitive advantage of concept maps results from combining visual and verbal modes in externally representing knowledge. By using verbal labels for indicating the kind of relationship between nodes, the semantics of a spatial configuration of nodes may be comprehended and communicated more easily due to advantages resulting from dual coding of visual and verbal stimuli.

\section{Activity as a Scaffolding Strategy}

Scaffolding has been used to facilitate learners in coping with the challenges of complex problems solving. Scaffolds can be defined as temporary supports provided by the teacher or another student to help Learners Bridge the gap between their current abilities and the intended goal [5], [6].

In order to learn, learners need to attend to and process elements of new information, establish key connections between them, integrate them with available knowledge base, and build new or modified knowledge structures. These processes occur in WM and inevitably impose a cognitive load. However this load is essential for learning.

According to Vygotsky, learners should be guided or scaffolded by a "more capable peer" to solve a problems or carry out a task that would be beyond what they could accomplish independently. Scaffolding can help learners accomplish more than what they could accomplish by themselves by providing them with conceptual, metacognitive, procedural, and strategic support that can assist them as they approach and solve ill-structured problems [5]-[8].

The activity system as a whole remains the principal, holistic object of analysis in mobile learning. It is represented by the activity triangle (Fig. 3).

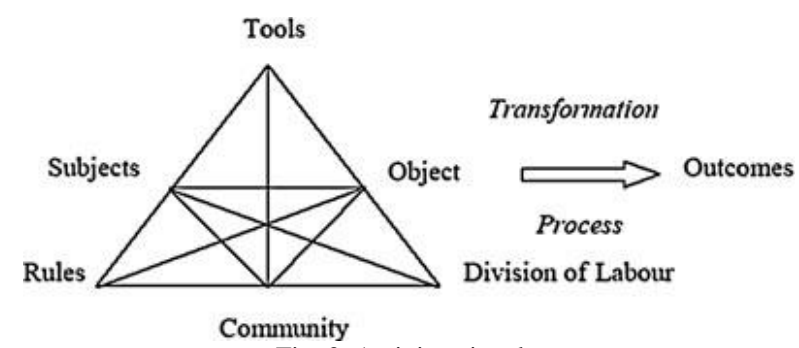

Fig. 3. Activity triangle.

Activities have been found to be effective in facilitating ill-structured problems-solving processes, including problems representation, developing solutions, making justifications, and monitoring and evaluation .

- Activity can support learners in representing problems by directing their attention to important characteristics, 
helping them identify relevant information, eliciting explanation, and activating their prior knowledge.

- Activity can help learners develop solutions by making connections to their existing knowledge and directing their attention to identifying goals and solution constraints.

- Activity can support learners in developing justifications for their solutions by helping them articulate their ideas.

- Activity can support learners as they organize, monitor, and evaluate by making their thinking and learning process explicit.

\section{Resources: Advance Organizers}

The concept of advance organizers was first formally introduced by Ausubel as a vehicle for testing the theory of cognitive subsumption. Advance organizers are material introduced in advance of the material to be learned and are presented at a higher level of abstraction, generality, and inclusiveness. Advance organizers will promote connections between prior knowledge and the to-be-learned material.

New materials may be incorporated into cognitive structures as long as it is subsumable under relevant existing concepts. Thus, unfamiliar but meaningful verbal material may be learned and recalled with less difficulty by the advance introduction of relevant subsuming concepts. This is demonstrated via the use of what we called advance organizers. There are two types of advance organizers: expository and comparative.

- Expository advance organizers are appropriate with completely unfamiliar material. They provide relevant proximate subsumes. These subsumes bear a superordinate relationship to the new material and provide ideational anchorage in terms that are familiar to the learner.

- Comparative advance organizers are used with relatively familiar material. They facilitate integration of new ideas with basically similar concepts in the learner's cognitive structure. The intent of a comparative advance organizer is to provide information that enhances discriminability between new and existing ideas which are essentially different but confusable similar.

\section{Conclusions}

Ill-structured problems for e-learning is no clear solution path and there are multiple possible solutions. Learners need to define the problems, generate and pursue learning issues, and develop a possible solution to the problems. It provides method to promote ill-structured problems solving.

Using maps may solve - at least partially - problems associated with visualizing information structures to make information more salient to users by providing semantically comprehensible access. Activities have been found to be effective in facilitating ill-structured problems-solving processes. Metacognition, which involves both knowledge and regulation of cognition, is necessary for solving ill-structured problems. Advance organizers will promote connections between prior knowledge and the to-be-learned material. It is necessary to reduce extraneous cognitive load caused by interactivity. Learner cognitive characteristics should be taken into account rather than relying rigidly on some fixed principles. The aim is to transfer learner behavioral activity into cognitive interactivity that generates essential cognitive processing relevant to knowledge construction without increasing non-essential extraneous processing load.

\section{REFERENCES}

[1] C. Chin and L. Chia, "Problems-based learning: Using ill-structured problemss in biology project work," Science Education, vol. 90, no. 1, pp. 44-67, 2005

[2] I. L. Chicago et al., "Cognitive requirements for learning with open-ended learning environments," Educational Technology Research and Development, vol. 48, no. 3, pp. 61-78, 2000.

[3] M. Weideman and W. Kritzinger, "Concept Mapping - a proposed theoretical model for implementation as a knowledge repository," Working paper from the "ICT in Higher Education" research project, University of Western Cape - South Africa, 2003.

[4] E. A. Kyza and D. C. Edelson, "Reflective inquiry: What it is and how can software scaffolds help," presented at the Annual Meeting of the American Educational Research Association, April 2003.

[5] R. Azevedo and M. J. Jacobson, "Advances in scaffolding learning with hypertext and hypermedia: A summary and critical analysis," Educational Technology Research and Development, vol. 56, no. 1, pp. 93-100, 2008.

[6] S. M. Land and C. Zembal-Saul, "Scaffolding reflection and articulation of scientific explanations in a data-rich, project-based learning environment: An investigation of progress portfolio," Educational Technology Research and Development, vol. 51, no. 4, pp. 65-84, 2003.

[7] R. Azevedo, D. C. Moos, J. A. Greene, F. I. Winters, and J. G. Cromley, "Why is externallyregulated learning more effective than self-regulated learning with hypermedia?" Educational Technology Research and Development, vol. 56, no. 1, pp. 45-72, 2008.

[8] A. C. Graesser, D. S. McNamara, and K. VanLehn, "Scaffolding deep comprehension strategies through Point\&Query," AutoTutor, and iSTART. Educational Psychologist, vol. 40, pp. 225-234, 2005.

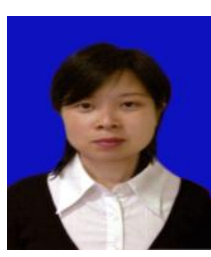

Jianzhen Chen is a doctoral student of Beijing Institute of Technology, and now she is a teacher in Tianjin University of Technology and Education. Her research interests include information technology, and vocational education.

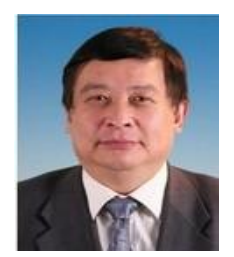

Xiaoping $\mathbf{L i}$ is a professor of Beijing Institute of Technology. His recent articles can be found in Studies in Computational Intelligence, Emerging Computation and Information technologies for Education, Digital Content, Multimedia Technology and its Applications, and other Chinese distance education journals. 